\title{
Influencia de la comunicación publicitaria en el consumo de alcohol
}

\author{
Carmen LóPEZ-SÁNCHEZ \\ Universidad de Alicante \\ mc.lopez@ua.es \\ José A. García Del Castillo \\ Universidad Miguel Hernández \\ jagr@umh.es \\ Mónica GÁzQuez Pertusa \\ Universidad Miguer Hernández \\ mgazquez@umh.es
}

\begin{abstract}
Resumen:
La literatura científica aduce que a mayor exposición de mensajes publicitarios de bebidas alcohólicas, mayor probabilidad de que estas sean consumidas. Método. La muestra constó de 437 estudiantes universitarios. Los objetivos se centraron en analizar la relación entre mensaje publicitario y consumo. Resultados. Existe relación entre publicidad y consumo, dado que el consumo de los jóvenes coincide con el recuerdo de las campañas en cuanto al tipo de bebida consumida. Conclusiones. Observamos que la publicidad parece ser un instrumento de influencia al consumo de alcohol.
\end{abstract}

Palabras Clave: Publicidad - Influencia - Consumo de alcohol - Estudiantes universitarios.

\section{Advertising communication influence in alcohol consumption}

\begin{abstract}
:
Scientific literature says the greater exposure to advertising material about alcohol drinks leads to a greater consumption. Method. The sample contained 437 university students. The objectives were focused on analyze the relationship between advertising message and consumption. Results. A relationship between advertising and consumption was found, as the youth consumption coincides with the advertising campaign remembrance in terms of kind of drink consumed. Conclusions. We note the advertising appears to be an influential instrument for alcohol consumption.
\end{abstract}

Keywords: Advertising - Influence - Alcohol consumption - University Students

\section{Referencia normalizada:}

López Sánchez, C.; García del Castillo, J. A.; Gázquez Pertusa, M. (2013) Influencia de la comunicación publicitaria en el consumo de alcohol. Historia y Comunicación Social. Vol. 18 No Especial Octubre. Págs. 639-655.

Sumario: 1. Introducción. 2. Método. 2.1. Muestra. 2.2. Instrumentos. 2.3. Diseño. 2.4. Procedimiento. 3. Resultados. 3.1. Campaña publicitaria más recordada. 3.2. Consumo de alcohol. 3.3. Relación entre la publicidad y consumo de alcohol. 3.4. Actitudes hacia el alcohol. 4. Conclusiones. 5. Referencias. 


\section{Introducción}

El estudio de las adicciones implica la participación de muchas disciplinas, ya que no cuenta con un cuerpo teórico propio y necesita recurrir a diferentes ramas del conocimiento. El término droga y su conceptualización conlleva connotaciones contradictorias, ya que por una parte son beneficiosas para la salud si se administran adecuadamente ante procesos de enfermedad y por otra son destructivas si se opta por un consumo recreativo indiscriminado. La complejidad de su estudio es otro indicador fiable de que necesitamos analizar secuencialmente todos los aspectos relacionados con el comportamiento de consumo, porque los procesos de influencia pueden alojarse en diferentes vertientes de la conducta. En principio, es indistinto que estudiemos sustancias legales o ilegales, porque los mecanismos de funcionamiento de la adicción son similares.

En nuestra cultura las drogas legales que tienen mayor incidencia y generan más problemas son el tabaco y el alcohol. De hecho las tasas de consumo de estas sustancias han mantenido unos niveles altos entre la población a lo largo de los años con una leve tendencia a la baja, a pesar de los programas preventivos que se han ido poniendo en marcha. La prevalencia de consumo diario de tabaco en el año 1997 era del 34,9\% y en el 2011 es del 30,4\%. En cuanto al alcohol, la prevalencia de consumo en los últimos 30 días en el año 1997 era del 64\% y en el año 2011 del 62,3\% (Plan Nacional sobre Drogas, 2011). Los cambios más sustanciales para regular los consumos de estas sustancias han pivotado en la legislación, la prevención y la promoción de la salud. Una de las cuestiones primordiales que abordó la legislación fue el desarrollo de una normativa más restrictiva para la publicidad de estas sustancias, siendo en el caso del tabaco mucho más coercitiva. El alcohol sigue siendo una sustancia más protegida o privilegiada en el tratamiento legislativo y popular, contando todavía con mayor flexibilidad para su promoción.

La comunicación publicitaria ha tenido que desplegar todo su ingenio para poder bordear la legislación y seguir publicitando estas sustancias. Podemos analizar algunas formas de publicidad que consiguen llegar al público objetivo, sin necesidad de hacerlo de una forma explícita o mediante los clásicos anuncios y campañas publicitarias.

Una estrategia de uso común, que tiene una presencia alta en nuestros medios, es la que podíamos denominar "publicidad noticiada". Medios como la radio o la televisión, que cuentan con una amplia programación de informativos, pueden incluir noticias relacionadas con el tabaco o el alcohol fácilmente con independencia de que tengan más o menos fundamento o repercusión social. De hecho si hacemos una búsqueda en el Google Trends encontramos más de 200 noticias con el término "noticias de tabaco" y más de 450 si introducimos en término "noticias de alcohol", desde enero a septiembre del año 2013 en España. Posiblemente las empresas alcoholeras y tabaqueras no sean las que propongan estas noticias ex profeso, pero sí pueden ejercer influencia o tratar de que salten noticias a los medios utilizando estrategias de marketing sofisticadas. En principio cualquier noticia sobre sustancias adictivas 
puede generar inducción al consumo, dado que la mera información ya supone una forma de promoción aunque el mensaje contenga elementos negativos (García del Castillo, López-Sánchez y García del Castillo-López, 2009).

Una segunda estrategia ampliamente utilizada en televisión es el llamado brand placement, una forma encubierta de introducir productos en las series de televisión, en el cine y en cualquier soporte de ficción. Muchas series españolas construyen los guiones incluyendo escenarios que les permitan introducir este tipo de publicidad, como un bar, una cafetería, una farmacia, etc. Cualquier producto se puede publicitar con esta estrategia, pero para aquellos que tienen prohibida la publicidad expresamente es una fórmula impecable, porque una máquina que suministra tabaco se puede camuflar perfectamente en cualquier rincón de la escena o la exposición de botellas de alcohol de alta graduación en los estantes del bar (Del Pino, López-Sánchez y García del Castillo, 2009; Del Pino y Olivares, 2006).

Una tercera estrategia algo más sofisticada y vanguardista es el branded content. En este formato la publicidad es el motivo principal de su razón de ser, es decir, que se diseña ex profeso un programa para publicitar determinada marca y de esta manera el que visiona el programa puede hacer un seguimiento fácil de la marca a través del mismo.

Es incuestionable que si se sigue introduciendo publicidad de bebidas alcohólicas en los medios es porque tiene una repercusión en el consumo, otra cuestión diferente es si la propia publicidad es capaz de generar una base de influencia suficiente como para promocionarlo o aumentarlo en poblaciones que previamente no consumen alcohol. La publicidad cuenta con un factor que en principio es dudoso pero que acaba teniendo un efecto positivo en el usuario, es la credibilidad del mensaje. Por increíble que pueda llegar a ser el mensaje publicitario, a base de reiterarlo y repetirlo consigue que la credibilidad del usuario aumente y lo persuada de comprar o consumir un determinado producto, desde el agua adelgazante hasta el crecepelo (Del Pino y Olivares, 2006; García del Castillo, 2006).

Según Sánchez Pardo (2006), se puede considerar que la publicidad es un factor de riesgo en la iniciación al consumo de bebidas alcohólicas en la población joven. Aunque la literatura científica no ha demostrado fehacientemente la relación tácita entre publicidad y consumo de alcohol, la tendencia de los estudios revisados es poner de relieve esta asociación (Anderson, Bruijn, Angus, Gordon y Hastings, 2009; Bryden, Roberts, McKee y Petticrew, 2012; Hanewinkelet al., 2012; Jones y Magee, 2011; Laaoula, 2012; Smith y Foxcroft, 2009). La probabilidad de iniciarse en el consumo de alcohol aumenta ante una mayor exposición a mensajes publicitarios de bebidas alcohólicas, así como el aumento del consumo en los ya iniciados (Montes-Santiago, Álvarez y Baz, 2007; Thomsen y Rekve, 2006; van Daleny Kuunders, 2006). Únicamente falta establecer relaciones causales que demuestren que esta relación publicidad de alcohol-consumo de alcohol se establece como causa-efecto. No obstante estudios como los de Caicedo, Fornero, Pauolocci y Godoy (2012) 
concluyen que la exposición a la publicidad de bebidas alcohólicas no modifica las cogniciones implícitas asociadas al alcohol, como las creencias y las actitudes.

El objetivo de este trabajo consiste en analizar el recuerdo de las campañas publicitarias sobre bebidas alcohólicas de los estudiantes universitarios y la relación con el consumo de alcohol, las actitudes hacia su consumo y las principales motivaciones para beber alcohol.

\section{Método}

\subsection{Muestra}

Participaron en el estudio 437 estudiantes universitarios. Del total de la muestra un $32 \%$ eran hombres y un $68 \%$ mujeres, presentando una edad media de 21,54 (desv. típ. 4,715). Los 438 sujetos respondieron a un cuestionario on-line elaborado al efecto (Tabla 1).

Tabla 1. Características de identificación de la muestra.

\begin{tabular}{|l|l|}
\hline & $\mathrm{N}=115$ \\
\hline Edad Media (DT) & $21,54(4,720)$ \\
\hline Sexo & \\
\hline$\%$ Hombres (n) & $31,6(138)$ \\
\hline$\%$ Mujeres (n) & $68,4(299)$ \\
\hline Universidad & \\
\hline Univ. Alicante & $60,4(264)$ \\
\hline Univ. Miguel Hernández & $29,3(128)$ \\
\hline Univ. de Murcia & $8,7(38)$ \\
\hline Univ. Carlos III de Madrid & $1,6(7)$ \\
\hline Titulación & \\
\hline Publicidad & $51,2(222)$ \\
\hline Terapia Ocupacional & $2,8(12)$ \\
\hline Trabajo Social & $7,4(32)$ \\
\hline Psicología & $16,8(73)$ \\
\hline Periodismo & $17,7(77)$ \\
\hline Máster en Comunicación e Industrias Creativas & $1,4(6)$ \\
\hline Comunicación Audiovisual & $1,6(7)$ \\
\hline Criminología & $0,2(2)$ \\
\hline Ingeniería e Informática & $0,2(2)$ \\
\hline Química & $0,5(2)$ \\
\hline Ingeniero de Telecomunicaciones & $0,2(1)$ \\
\hline Curso & \\
\hline Primero & $28,1(123)$ \\
\hline
\end{tabular}




\begin{tabular}{|l|l|}
\hline Segundo & $42,8(187)$ \\
\hline Tercero & $13,3(58)$ \\
\hline Cuarto & $10,8(47)$ \\
\hline Quinto & $5(22)$ \\
\hline
\end{tabular}

\subsection{Instrumentos}

Para recabar la información se empleó un Cuestionario de Publicidad y Alcohol (PUBA), elaborado al efecto, que sondeaba cuestiones referentes a la publicidad, el consumo de alcohol y la valoración que los sujetos mantienen sobre la influencia de la publicidad y el consumo de alcohol.

El cuestionario estaba formado por preguntas de respuesta múltiple y preguntas abiertas agrupadas en tres grandes bloques:

1. Campaña publicitaria más recordada por los sujetos, dónde se recabó información sobre la marca, el producto, los medios de comunicación, el número de veces que la habían visto y los elementos de la campaña que más impacto les generaron.

2. Consumo de Alcohol, se recogieron indicadores del consumo de alcohol como: la frecuencia de consumo general y desglosada en función del consumo en días laborables y no laborables, la frecuencia semanal en función del tipo de bebida, los episodios de embriaguez, la cantidad desglosada según el tipo de bebida y los motivos para beber. Por último a los sujetos se les solicitaba realizar una estimación subjetiva de su consumo global de alcohol en una escala graduada de 0 a 10.

3. Relación entre la publicidad y el consumo de alcohol, donde se sondeaban las creencias subjetivas sobre la influencia que la publicidad ejerce en el consumo de alcohol, y las actitudes y valores asociados al consumo. Para ello este bloque se desglosó en dos apartados diferenciados en función de si la influencia se ejercía sobre uno mismo o sobre los demás.

A su vez y con objeto de recabar las actitudes que los sujetos mantienen hacia el consumo de alcohol se administró la Escala de Actitudes hacia el alcohol (ESACAL) de García-Rodríguez y López-Sánchez (2001). Compuesta de 13 ítems tipo Likert (1-5), que evalúa las actitudes hacia el consumo de alcohol. La escala muestra correlaciones test-retest significativas $\mathrm{r}=, 638 ; \mathrm{p} \leq .01$ y se compone de tres factores, con saturaciones mayores a 0,40 y un total de varianza explicada de 64,09\%: Factor 1 : Disposición actitudinal desfavorable al consumo; Factor2: Índice de desagrado; y Factor3: Predisposición a la acción contra el consumo. Una puntuación elevada en cada uno de los factores indica una actitud contraria al consumo de alcohol y viceversa.

\subsection{Diseño}

Se utilizó un diseño de corte transversal y descriptivo. 


\subsection{Procedimiento}

Se solicitó a los sujetos su participación voluntaria y anónima en el estudio. Se les proporcionó una dirección web donde estaba alojado el cuestionario. En el último campo del cuestionario se les solicitaba a los participantes que introdujeran una clave de identificación para verificar su participación.

\section{Resultados}

\subsection{Campaña publicitaria más recordada.}

Cuando se analizan la información referente a la campaña publicitaria más recordada se destacan varios aspectos relevantes.

En primer lugar, el producto anunciado en la campaña publicitaria más recordada, por la mayoría de los sujetos de la muestra, es la cerveza Heineken $(14,1 \%)$ seguida de Bacardi Mojito (12,05\%) y Martini $(8,47 \%)$. En la tabla 2 se detallan los porcentajes obtenidos para el resto de productos distinguidos por los encuestados.

Tabla 2. Producto anunciado en la campaña publicitaria más recordada.

\begin{tabular}{|l|c|c|}
\hline Marca & \% & n \\
\hline Bacardi & 1,95 & 6 \\
\hline Martini & 8,47 & 26 \\
\hline Ron Barceló & 4,56 & 14 \\
\hline Estrella Damm & 5,54 & 17 \\
\hline Heineken & 14,01 & 43 \\
\hline Cruzcampo & 7,82 & 24 \\
\hline Mixta & 6,84 & 21 \\
\hline San Miguel & 2,61 & 8 \\
\hline Bacardi Mojito & 12,05 & 37 \\
\hline Crema Caballero & 2,61 & 8 \\
\hline Cacique Mojito & 5,54 & 17 \\
\hline Ron Cacique & 1,95 & 6 \\
\hline Baileys & 3,58 & 11 \\
\hline Brugal & 1,95 & 6 \\
\hline Otros & 20,52 & 63 \\
\hline Total & & 307 \\
\hline
\end{tabular}


En consonancia con el producto, como se detalla en la tabla 3, las marcas más recordadas mediante la campaña publicitaria son Heineken y Bacardi $(14,29)$, seguidas por Martini $(13,82)$, Cruzcampo $(7,73)$ y Estrella Damm $(7,03)$.

Tabla 3. Marca de la campaña publicitaria más recordada.

\begin{tabular}{|l|l|l|}
\hline Marca & \% & n \\
\hline Bacardi & 14,29 & 61 \\
\hline Martini & 13,82 & 59 \\
\hline Eristoff & 1,64 & 7 \\
\hline Ballantines & 1,87 & 8 \\
\hline Barceló & 5,39 & 23 \\
\hline Estrella Damm & 7,03 & 30 \\
\hline Heineken & 14,29 & 61 \\
\hline Cruzcampo & 7,73 & 33 \\
\hline Mixta & 1,87 & 8 \\
\hline San Miguel & 3,51 & 15 \\
\hline Caballero & 1,87 & 8 \\
\hline Cacique & 5,62 & 24 \\
\hline Mahou & 3,04 & 13 \\
\hline Malibú & 1,64 & 7 \\
\hline Baileys & 2,11 & 9 \\
\hline Brugal & 1,87 & 8 \\
\hline Otros & 12,41 & 53 \\
\hline Total & & 427 \\
\hline & & \\
\hline & & \\
\hline
\end{tabular}

El tipo de bebida más frecuentemente publicitado en la campaña se corresponde con bebidas alcohólicas de baja graduación, con un porcentaje igual a 57,7\%, seguido por las bebidas de alta graduación con un $28,8 \%$ (Tabla 4). 
Tabla 4. Tipo de bebida anunciada en la campaña publicitaria más recordada.

\begin{tabular}{|l|l|l|}
\hline & $\mathbf{\%}$ & $\mathbf{n}$ \\
\hline $\begin{array}{l}\text { Bebidas alcohólicas de baja graduación (cerveza, vino, combinados } \\
\text { embotellados, etc.). }\end{array}$ & 57,7 & 252 \\
\hline Bebidas alcohólicas de media graduación (vermut, pacharán, jerez,...). & 13,3 & 58 \\
\hline Bebidas alcohólicas de alta graduación (ginebra, whisky, ron, etc.). & 28,8 & 126 \\
\hline Total & & 436 \\
\hline
\end{tabular}

Por otro lado, la campaña publicitaria se ha conocido principalmente a través de la televisión (89,2\%), seguido por internet $(16,2 \%)$ y la publicidad exterior como vallas, transportes, lonas de fachadas, cabinas telefónicas, mobiliario urbano, etc. (16\%). Y, además, un $68,8 \%$ de los sujetos de la muestra han visto la campaña más de 10 veces (Véanse tablas 5 y 6 ).

Tabla 5. Medio a través del cual se conoce la campaña.

\begin{tabular}{|l|l|l|}
\hline & SI \% (n) & NO \% (n) \\
\hline Televisión & $89,2(390)$ & $10,8(47)$ \\
\hline Publicidad exterior & $16(70)$ & $84(367)$ \\
\hline Medios impresos & $12,5(55)$ & $87,4(382)$ \\
\hline Radio & $6,2(27)$ & $93,8(410)$ \\
\hline Cine & $0,7(3)$ & $99,3(434)$ \\
\hline Internet & $16,2(71)$ & $83,8(366)$ \\
\hline
\end{tabular}

Tabla 6. Veces que han visto la campaña.

\begin{tabular}{|l|l|l|}
\hline & \% & (n) \\
\hline Entre 1 y 5 veces. & 7,8 & 34 \\
\hline Entre 6 y 10 veces. & 23,4 & 102 \\
\hline Más de 10 veces. & 68,8 & 300 \\
\hline
\end{tabular}

Con respecto, a la valoración subjetiva sobre el impacto de los diferentes componentes de la campaña, en una escala de 0 a 10 , los sujetos en promedio han otorgado en torno a 7 puntos aspectos como la ambientación, la creatividad y/o originalidad y la música (Tabla 7). 
Tabla 7. Valoración subjetiva del impacto de cada componente de la campaña.

\begin{tabular}{|l|l|l|}
\hline & Media & Desv. típ. \\
\hline El eslogan. & 5,66 & 2,742 \\
\hline La música. & 6,81 & 2,986 \\
\hline La historia que cuenta. & 6,26 & 2,797 \\
\hline Los colores. & 6,41 & 2,314 \\
\hline Los personajes. & 6,41 & 2,549 \\
\hline Las emociones que me despierta. & 6,65 & 2,463 \\
\hline El mensaje que lanza. & 6,23 & 2,526 \\
\hline Su creatividad/originalidad. & 6,92 & 2,347 \\
\hline El diseño gráfico. & 5,79 & 2,422 \\
\hline La ambientación. & 7,23 & 2,057 \\
\hline El humor que me provoca. & 6,09 & 2,968 \\
\hline & & \\
\hline
\end{tabular}

\subsection{Consumo de alcohol}

En promedio, en una escala de 0 a 10 puntos, los sujetos encuestados sitúan su consumo de alcohol en torno a 4 puntos $(\mathrm{dt} .=2,21)$. Aunque tales estimaciones han oscilado entre un mínimo de 0 puntos y un máximo de 10 (Tabla 8).

Tabla 8. Estimación subjetiva del consumo de alcohol propio.

\begin{tabular}{|c|c|c|}
\hline & \% & $\mathbf{n}$ \\
\hline 0 & 8,2 & 36 \\
\hline 1 & 9,2 & 40 \\
\hline 2 & 12,6 & 55 \\
\hline 3 & 16,7 & 73 \\
\hline 4 & 12,1 & 53 \\
\hline 5 & 13,7 & 60 \\
\hline 6 & 15,1 & 66 \\
\hline 7 & 9,8 & 43 \\
\hline 8 & 1,8 & 8 \\
\hline 9 & 0,2 & 1 \\
\hline 10 & 0,5 & 2 \\
\hline Total & & 437 \\
\hline
\end{tabular}


Por otra parte, un $42 \%$ declara consumir alcohol semanalmente, mientras que aproximadamente un $30 \%$ consume mensualmente y un $23 \%$ rara vez consume alcohol (Tabla 9).

Tabla 9. Consumo de alcohol.

\begin{tabular}{|l|l|l|}
\hline & $\mathbf{\%}$ & $\mathbf{n}$ \\
\hline Nunca & 5,0 & 22 \\
\hline Rara vez & 22,9 & 100 \\
\hline Mensualmente & 29,7 & 130 \\
\hline Semanalmente. & 42,1 & 184 \\
\hline A diario & 0,1 & 1 \\
\hline Total & & 437 \\
\hline
\end{tabular}

Con respecto a la frecuencia general, la mayoría de los encuestados afirma beber alcohol sólo los fines de semana (46,7\%) y un $32,7 \%$ en fiestas y reuniones (Tabla $10)$.

Tabla 10. Frecuencia de consumo de alcohol.

\begin{tabular}{|l|l|l|}
\hline & $\mathbf{\%}$ & $\mathbf{n}$ \\
\hline No bebo & 5,3 & 23 \\
\hline Muy rara vez & 13,3 & 58 \\
\hline Sólo en celebraciones: fiestas y reuniones & 32,7 & 143 \\
\hline Sólo fines de semana & 46,7 & 204 \\
\hline Sólo en las comidas & 1,1 & 5 \\
\hline Todos los días &, 9 & 4 \\
\hline Total & & 437 \\
\hline
\end{tabular}

Cuando se analiza la frecuencia de consumo diferenciando entre días laborables y fines de semana y/o festivos, se observa que lo más representativo es no beber entre semana $(59,3 \%)$. Sin embargo, la frecuencia más común los fines de semana y festivos es beber a veces $(46,5 \%)$ (Tabla 11$)$. 
Tabla 11. Frecuencia en función de días laborables o fines de semana.

\begin{tabular}{|l|c|c|c|c|c|}
\hline & $\begin{array}{c}\text { Nunca } \\
\mathbf{\%}(\mathbf{n})\end{array}$ & $\begin{array}{c}\text { A veces } \\
\mathbf{\%}(\mathbf{n})\end{array}$ & $\begin{array}{c}\text { Normalmente } \\
\mathbf{\%}(\mathbf{n})\end{array}$ & $\begin{array}{c}\text { Casi siempre } \\
\mathbf{\%}(\mathbf{n})\end{array}$ & $\begin{array}{c}\text { Siempre } \\
\mathbf{\%}(\mathbf{n})\end{array}$ \\
\hline $\begin{array}{l}\text { Entre semana } \\
\begin{array}{l}\text { Fines de } \\
\text { semana }\end{array}\end{array}$ & $59,3(259)$ & $38,2(167)$ & $2,1(9)$ & $0,5(2)$ & $0(0)$ \\
\hline
\end{tabular}

En referencia a la cantidad de consumiciones de alcohol (ver tabla 12), los sujetos consumen en promedio semanalmente entre 1 y 5 cañas, entre 1 y 5 copas de combinados de alta graduación y a lo largo de su vida se han emborrachado entre 2 y 6 veces. Con respecto al consumo de vino, chupitos y combinados de baja y media graduación la tendencia central se sitúa en la categoría nada. La distribución de los porcentajes obtenidos en el número de consumiciones según el tipo de bebida se muestra en la tabla 13.

Tabla 12. Cantidad de consumiciones de alcohol semanalmente. Estadísticos descriptivos.

\begin{tabular}{|l|c|c|}
\hline & Mediana & $\begin{array}{c}\text { Amplitud } \\
\text { Semi-intercuartil }\end{array}$ \\
\hline Cerveza & Entre 1 y 5 cañas & 0,5 \\
Vino & Nada & 0 \\
Combinados - Graduación Baja & Nada & 0 \\
Combinados - Graduación Media & Nada & 0 \\
Combinados - Graduación Alta & Entre 1 y 5 copas. & 0,5 \\
Chupitos & Nada & 0,5 \\
Episodios de embriaguez & Entre 2 y 6 veces & 0,5 \\
\hline
\end{tabular}

Tabla 13. Cantidad de consumiciones de alcohol en función del tipo de bebida.

\begin{tabular}{|l|l|l|l|l|l|}
\hline & Nada & $\mathbf{1 - 5}$ copas & $\mathbf{6 - 9}$ copas & $\mathbf{1 0 - 1 5}$ copas & $\mathbf{1 6 - 2 0}$ copas \\
\hline Cerveza & 46,9 & 44,6 & 6,2 & 2,3 & 0 \\
Vino & 81,9 & 16,0 & 1,1 &, 9 & \\
$\begin{array}{l}\text { Combinados - } \\
\text { Graduación Baja }\end{array}$ & 85,8 & 14,0 & 0 &, 2 & 0 \\
$\begin{array}{l}\text { Combinados - } \\
\text { Graduación Media } \\
\begin{array}{l}\text { Combinados - } \\
\text { Graduación Alta }\end{array}\end{array}$ & 92,0 & 7,6 &, 2 & 0 &, 2 \\
\hline
\end{tabular}




\begin{tabular}{|l|l|l|l|l|l|}
\hline & Nada & $\begin{array}{l}\mathbf{1 - 2} \\
\text { chupitos }\end{array}$ & $\begin{array}{l}\mathbf{3 - 4} \\
\text { chupitos }\end{array}$ & $\mathbf{5 - 6}$ chupitos & $\begin{array}{l}\mathbf{7 - 8} \\
\text { chupitos }\end{array}$ \\
\hline Chupitos & 55,6 & 34,6 & 7,8 & 1,6 &, 5 \\
\hline & Nunca & Una vez & $2-6$ veces & Más de 6 & He perdido \\
& & & veces & la cuenta \\
\hline $\mathrm{N}^{\text {o de embriagueces }}$ & 11,9 & 10,1 & 28,6 & 22,9 & 26,5 \\
\hline
\end{tabular}

Cuando se analizan los motivos más señalados por los que los sujetos de la muestra consumen alcohol, como se aprecia en la tabla 14, la mayoría de los sujetos bebe porque les gusta coger el punto $(47,8 \%)$, para divertirse $(44,4 \%)$, porque les gusta su sabor $(39,6 \%)$, para desinhibirse $(20,8 \%)$, porque les refresca $(16,5 \%)$ y para relacionarse mejor $(13,5 \%)$.

Tabla 14. Principales motivos para consumir alcohol.

\begin{tabular}{|l|c|c|}
\hline & $\begin{array}{c}\text { SI } \\
\text { (n) }\end{array}$ & $\begin{array}{c}\text { NO } \\
\text { (n) }\end{array}$ \\
\hline No bebo & $10,3(45)$ & $89,7(392)$ \\
\hline Porque me gusta "coger el punto". & $47,8(209)$ & $52,2(228)$ \\
\hline Porque lo hacen mis amigos. & $4,6(20)$ & $95,4(417)$ \\
\hline Para relajarme. & $8,9(39)$ & $91,1(398)$ \\
\hline Para relacionarme mejor. & $13,5(59)$ & $86,5(378)$ \\
\hline Para superar problemas. & $1,1(5)$ & $98,9(432)$ \\
\hline Para desinhibirme. & $20,8(91)$ & $79,2(346)$ \\
\hline Para sentirme mejor. & $3,7(16)$ & $96,3(421)$ \\
\hline Para aliviar el aburrimiento. & $7,8(34)$ & $92,2(403)$ \\
\hline Para divertirme. & $44,4(194)$ & $55,6(243)$ \\
\hline Porque me gusta su sabor. & $39,6(173)$ & $60,4(264)$ \\
\hline Para mejorar mis relaciones sexuales. & $1,4(6)$ & $98,6(431)$ \\
\hline Para levantar mi estado de ánimo. & $8,9(39)$ & $91,1(398)$ \\
\hline Para sentirme más integrado con mi grupo. & $4,3(19)$ & $95,7(418)$ \\
\hline Porque me refresca. & $16,5(72)$ & $83,5(365)$ \\
\hline Porque no se puede hacer otra cosa durante el ocio. & $2,1(9)$ & $97,9(428)$ \\
\hline Porque los lugares de ocio son para consumir alcohol. & $3,2(14)$ & $96,8(423)$ \\
\hline
\end{tabular}


Por otro lado, el ron es la bebida preferida para un $27,8 \%$ de los sujetos, seguida por la cerveza $(22,9 \%)$ y el vodka $(17 \%)$. El resto de porcentajes se detalla en la tabla 15.

Tabla 15. Tipo de bebida favorita

\begin{tabular}{|l|l|l|}
\hline Tipo de Bebida & $\mathbf{\%}$ & $\mathbf{n}$ \\
\hline Cerveza & 22,9 & 100 \\
\hline Vino. & 6,0 & 26 \\
\hline Vodka. & 17,0 & 74 \\
\hline Ginebra. & 8,0 & 35 \\
\hline Ron. & 27,8 & 121 \\
\hline Whisky. & 5,5 & 24 \\
\hline Licores. & 2,5 & 11 \\
\hline Vermut. & 1,1 & 5 \\
\hline Botellines combinados. & 2,1 & 9 \\
\hline Coñac. &, 7 & 3 \\
\hline Champan. & 1,1 & 5 \\
\hline Ponche &, 5 & 2 \\
\hline Cócteles &, 7 & 3 \\
\hline Tequila &, 5 & 2 \\
\hline No bebo & 3,7 & 16 \\
\hline Total & & 436 \\
\hline
\end{tabular}

A su vez, la marca favorita de los encuestados es el Brugal (13\%), seguida por Heineken $(0,06 \%)$, Absolut $(0,07 \%)$, Smirnoff $(0,05)$ y Beefeater $(0,05 \%)$ (Tabla 16$)$.

Tabla 16. Marca preferida.

\begin{tabular}{|l|l|l|}
\hline & $\mathbf{\%}$ & $\mathbf{n}$ \\
\hline Bacardi & 0,03 & 12 \\
\hline Martini & 0,02 & 8 \\
\hline Eristoff Black & 0,02 & 8 \\
\hline Beefeater & 0,05 & 20 \\
\hline Ron Barceló & 0,04 & 15 \\
\hline Heineken & 0,06 & 25 \\
\hline Cacique Mojito & 0,02 & 9 \\
\hline Mahou & 0,03 & 12 \\
\hline Malibú & 0,01 & 6 \\
\hline
\end{tabular}




\begin{tabular}{|l|l|l|}
\hline Baileys & 0,01 & 6 \\
\hline Brugal & 0,13 & 54 \\
\hline Smirnoff vodka & 0,05 & 22 \\
\hline Absolut & 0,07 & 28 \\
\hline Budweiser & 0,02 & 7 \\
\hline J\&B & 0,02 & 7 \\
\hline Estrella de Levante & 0,02 & 8 \\
\hline Alhambra & 0,02 & 9 \\
\hline Coronita & 0,01 & 6 \\
\hline Negrita & 0,04 & 15 \\
\hline Matusalen & 0,02 & 7 \\
\hline Ninguna & 0,02 & 9 \\
\hline No bebo & 0,04 & 19 \\
\hline Otros & 0,26 & 112 \\
\hline Total & & 424 \\
\hline
\end{tabular}

3.3. Relación entre la publicidad y el consumo de alcohol

En referencia a las creencias subjetivas que los sujetos mantienen sobre la influencia que la publicidad ejerce en el consumo de alcohol, y las actitudes y valores asociados al consumo, los promedios registrados se detallan en la tabla 17. En promedio, se observa que los sujetos otorgan una mayor influencia a la publicidad, cuando se trata de los otros, que cuando se cuestionan la influencia que ejerce la publicidad sobre uno mismo.

Tabla 17. Relación entre la publicidad y el alcohol

\begin{tabular}{|l|l|l|l|l|}
\hline & Media & Desv. típ. & Mínimo & Máximo \\
\hline Influencia en primera persona (7-35) & 16,48 & 5,557 & 7 & 31 \\
\hline Influencia en tercera persona (7-35) & 24,11 & 4,470 & 9 & 35 \\
\hline & & & & \\
\hline
\end{tabular}

\subsection{Actitudes hacia el alcohol}

Total de la escala se detallan en la tabla 18. En general, se observa que los promedios registrados se sitúan por encima del punto medio de los rangos teóricos de los factores analizados.

Tabla 18. Actitudes hacia el alcohol 


\begin{tabular}{|l|c|c|c|c|c|}
\hline & Rango Teórico & Media & $\begin{array}{c}\text { Desv. } \\
\text { típ. }\end{array}$ & Mínimo & Máximo \\
\hline $\begin{array}{l}\text { Disposición actitudinal } \\
\text { contra el consumo }\end{array}$ & $6-30$ & 22,82 & 3,751 & 11 & 30 \\
\hline Índice Desagrado & $4-20$ & 8,80 & 3,060 & 4 & 20 \\
\hline $\begin{array}{l}\text { Predisposición a la acción } \\
\text { contra el consumo }\end{array}$ & $3-15$ & 12,00 & 1,901 & 4 & 15 \\
\hline Actitudes Total & $13-65$ & 43,63 & 6,654 & 23 & 64 \\
\hline & & & & & \\
\hline
\end{tabular}

\section{Conclusiones}

La mayor tasa de recuerdo de los estudiantes universitarios estudiados sobre las campañas publicitarias de bebidas alcohólicas, se encuentra en las bebidas de baja graduación como la cerveza y algunos licores o mezclas. Entre los de media graduación destaca el vermut. Fundamentalmente las marcas más recordadas son la cerveza Heineken, Cruzcampo y Estrella Damm, entre las de media graduación el vermut Martini y entre las de alta graduación el Ron Barceló.

En cuanto al medio por el que han entrado en contacto con la campaña publicitaria, destaca muy significativamente la televisión que aglutina más del $89 \%$ del total, seguida muy de lejos por Internet con más de un 16\% y por los medios impresos con un $12 \%$ aproximadamente. Es muy indicativo que la radio únicamente aglutine un $6,2 \%$, lo que indica que este medio no es muy seguido por esta población o bien que al carecer de imagen pierde impacto entre la población joven. Otro dato relevante es que más del $68 \%$ han visto la campaña más de 10 veces, lo que nos puede estar indicando que la estrategia de repetición es efectiva para que la tasa de recuerdo aumente y se mantenga la asociación marca-producto.

Es interesante destacar el análisis de los contenidos subjetivos de las campañas publicitarias de bebidas alcohólicas por parte de los jóvenes, ya que el mayor valor se lo dan a la ambientación del anuncio y la creatividad u originalidad del mismo, quedando en último lugar el eslogan, por detrás del mensaje o de los colores y personajes que se utilizan. Parece que la tendencia para que el impacto sea mayor está en relación con la forma de vender la bebida más que a la bebida en sí misma o lo que esta representa.

Hay que destacar que el consumo de alcohol de la población de jóvenes estudiada, coincide en gran medida con el recuerdo de las campañas publicitarias dado que el mayor consumo se concentra en la cerveza y las bebidas de alta graduación. Perciben subjetivamente que su consumo es bajo a pesar que más del $42 \%$ de la muestra bebe semanalmente y casi el 50\% afirma haberse emborrachado más de 6 veces en su vida o han perdido la cuenta. Los motivos por los que dicen beber se agrupan en torno al sabor de las bebidas, como estímulo para poder divertirse o para "coger el punto". 
Según la opinión de los jóvenes en relación con las campañas publicitarias, creen que en general ejercen una gran influencia sobre los demás, pero en menor medida sobre ellos mismos. No deja de ser paradójico que la tasa de influencia se perciba más alta cuando se valora a los iguales y sea significativamente más baja cuando la valoración es sobre uno mismo.

Podemos apuntar un perfil de la muestra estudiada que se configura como un joven estudiante universitario entre 18 y 25 años, que suele beber durante los fines de semana alcohol de baja y alta graduación, que ha visionado anuncios en televisión sobre campañas de alcohol en más de 10 ocasiones, que bebe fundamentalmente porque quiere coger el punto y divertirse, que se ha emborrachado más de 6 veces en su vida o ha perdido la cuenta.

\section{Referencias}

ANDERSON, P.; BRUIJN, A.; ANGUS, K.; GORDON, R.; HASTINGS, G. (2009). Impact of Alcohol Advertising and Media Exposure on Adolescent Alcohol Use: A Systematic Review of Longitudinal Studies. Alcohol \&Alcoholism, 44(3), 229-243.

BRYDEN, A.; ROBERTS, B.; MCKEE, M.; PETTICREW, M. (2012).A systematic review of the influence on alcohol use of community level availability and marketing of alcohol.Health \& Place, 18(2),349-357.

CAICEDO, E.E.; FORNERO, V.G.; PAUOLOCCI, C.; GODOY, J.C. (2012). La influencia de la publicidad de bebidas alcohólicas sobre las cogniciones implícitas asociadas al alcohol en estudiantes universitarios. Quaderns de Psicología, 14(1), 29-44.

DEL PINO, C.; OLIVARES, F. (2006). Brabdplacement: integración de marcas en la ficción audiovisual. Barcelona: Gedisa.

DEL PINO, C.; LÓPEZ-SÁNCHEZ, C.; GARCÍA DEL CASTILLO, J.A. (2009). El alcohol y el tabaco en los escenarios de ficción: el actor como prescriptor de conducta nocivas. En J.A. GARCÍA DEL CASTILLO; C. LÓPEZ-SÁNCHEZ (coords.). Medios de comunicación, publicidad y adicciones (205-224). Madrid: EDAF.

GARCÍA DEL CASTILLO, J.A. (2006). Credibilidad. Columna de Opinión: La pluma y el Diván. Diario La Verdad de Alicante, 10 de septiembre de 2006.

GARCÍA DEL CASTILLO, J.A.; LÓPEZ-SÁNCHEZ, C. (2009). Medios de comunicación, publicidad y adicciones. Madrid:EDAF.

GARCÍA DEL CASTILLO, J.A.; LÓPEZ-SÁNCHEZ, C.; GARCÍA DEL CASTILLO-LÓPEZ, A. (2009). El tratamiento informativo de las drogas en los medios de comunicación escrita y su repercusión en la prevención. En J.A. GARCÍA DEL CASTILLO; C. LÓPEZ-SÁNCHEZ (coords.). Medios de comunicación, publicidad y adicciones (23-46). Madrid: EDAF.

GARCÍA-RODRÍGUEZ, J.A.; LÓPEZ-SÁNCHEZ, C. (2001). Medida de las actitudes en la prevención de las drogodependencias. En J.A. García-Rodríguez y C. López-Sánchez (coord.). Manual de estudios sobre alcohol. Madrid: EDAF. 
HANEWINKEL, R.; SARGENT, J. D.; POELEN, E. A.; SCHOLTE, R.; FLOREK, E.; SWEETING, H.; ...Y MORGENSTERN, M. (2012). Alcohol consumption in movies and adolescent binge drinking in 6 European countries.Pediatrics, 129(4), 709-720.

JONES, S.C.; MAGEE, C.A. (2011). Exposure to Alcohol Advertising and Alcohol Consumption among Australian Adolescents.Alcohol and Alcoholism, 46(5), 630-637.

LAAOULA, O.N. (2012). Publicidad y Salud Comunitaria: Bebidas alcohólicas en el Prime Time. Revista Española de Comunicación en Salud, 3(2): 110-122.

MONTES-SANTIAGO, J.; ÁLVAREZ, M.L.; BAZ, A. (2007). Alcohol y publicidad en la prensa escrita en España. Anales de MedicinaInterna, 24(3), 109-112.

PLAN NACIONAL SOBRE DROGAS (2011). Encuesta sobre alcohol y drogas en población general en España (EDADES 2011-2012). Madrid: Observatorio Español sobre Drogas. $\mathrm{M}^{\mathrm{o}}$ de Sanidad, Servicios Sociales e Igualdad.

SÁNCHEZ PARDO, L. (2006). La publicidad de bebidas alcohólicas y tabaco. Madrid: Agencia Antidroga.

SMITH, L.A.; FOXCROFT, D.R. (2009).The effect of alcohol advertising, marketing and portrayal on drinking behaviour in young people: systematic review ofprospective cohort studies. BMC Public Health, 9, 51.

THOMSEN, S.R.; REKVE, D. (2006).The relationship between viewing US-produced television programs and intentions to drink alcohol among a group of Norwegian adolescents. Scandinavian Journal of Psychology, 47(1), 33-41.

VAN DALEN, W. E.; KUNDERS, M. M. A. P. (2006).Alcohol marketing and young people: an analysis of the current debate on regulation. Nordisk AlkoholochNarkotikatidskrift, 23(6), 415.

\section{Los autores}

Carmen López-Sánchez es Doctora en Psicología.y Profesora Titular de la Universidad de Alicante.

José A. García del Castillo es Doctor en Psicología. Catedrático de Escuela Universitaria. en la Universidad Miguel Hernández.

Mónica Gázquez Pertusa es Doctora en Psicología. Colaboradora de investigación. en la Universidad Miguel Hernández 